\title{
iMAP: an integrated bioinformatics and visualization pipeline for microbiome data analysis
}

Teresia M. Buza ${ }^{1,2^{*}} \mathbb{D}$, Triza Tonui ${ }^{3}$, Francesca Stomeo ${ }^{3,9}$, Christian Tiambo ${ }^{3}$, Robab Katani ${ }^{1,4}$, Megan Schilling ${ }^{1,5}$, Beatus Lyimo ${ }^{6}$, Paul Gwakisa ${ }^{7}$, Isabella M. Cattadori ${ }^{1,8}$, Joram Buza ${ }^{6}$ and Vivek Kapur ${ }^{1,4,5,6}$

\begin{abstract}
Background: One of the major challenges facing investigators in the microbiome field is turning large numbers of reads generated by next-generation sequencing (NGS) platforms into biological knowledge. Effective analytical workflows that guarantee reproducibility, repeatability, and result provenance are essential requirements of modern microbiome research. For nearly a decade, several state-of-the-art bioinformatics tools have been developed for understanding microbial communities living in a given sample. However, most of these tools are built with many functions that require an in-depth understanding of their implementation and the choice of additional tools for visualizing the final output. Furthermore, microbiome analysis can be time-consuming and may even require more advanced programming skills which some investigators may be lacking.
\end{abstract}

Results: We have developed a wrapper named iMAP (Integrated Microbiome Analysis Pipeline) to provide the microbiome research community with a user-friendly and portable tool that integrates bioinformatics analysis and data visualization. The iMAP tool wraps functionalities for metadata profiling, quality control of reads, sequence processing and classification, and diversity analysis of operational taxonomic units. This pipeline is also capable of generating web-based progress reports for enhancing an approach referred to as review-as-you-go (RAYG). For the most part, the profiling of microbial community is done using functionalities implemented in Mothur or QIIME2 platform. Also, it uses different R packages for graphics and R-markdown for generating progress reports. We have used a case study to demonstrate the application of the iMAP pipeline.

Conclusions: The iMAP pipeline integrates several functionalities for better identification of microbial communities present in a given sample. The pipeline performs in-depth quality control that guarantees high-quality results and accurate conclusions. The vibrant visuals produced by the pipeline facilitate a better understanding of the complex and multidimensional microbiome data. The integrated RAYG approach enables the generation of web-based reports, which provides the investigators with the intermediate output that can be reviewed progressively. The intensively analyzed case study set a model for microbiome data analysis.

Keywords: Microbiome bioinformatics, Microbiome data analysis, Microbiome data visualization, Microbial community, Bioinformatics pipeline, $16 \mathrm{~S}$ rRNA gene, Phylogenetic analysis, Phylogenetic annotation

\footnotetext{
* Correspondence: ndelly@gmail.com

'The Huck Institutes of the Life Sciences, Pennsylvania State University,

University Park, State College, PA, USA

${ }^{2}$ Department of Biochemistry and Molecular Biology, Pennsylvania State

University, University Park, State College, PA, USA

Full list of author information is available at the end of the article
}

(c) The Author(s). 2019 Open Access This article is distributed under the terms of the Creative Commons Attribution 4.0 International License (http://creativecommons.org/licenses/by/4.0/), which permits unrestricted use, distribution, and reproduction in any medium, provided you give appropriate credit to the original author(s) and the source, provide a link to the Creative Commons license, and indicate if changes were made. The Creative Commons Public Domain Dedication waiver (http://creativecommons.org/publicdomain/zero/1.0/) applies to the data made available in this article, unless otherwise stated. 


\section{Background}

Understanding the diversity of microbes living in a given sample is a crucial step that could lead to novel discoveries. The choice of bioinformatics methodology used for analyzing any microbiome dataset from pre-processing of the reads through the final step of the analysis is a key factor for gaining high-quality biological knowledge. Most of the available bioinformatics tools contain multiple functions and may require an in-depth knowledge of their implementation. In most cases, several tools are used independently to analyze a single microbiome dataset and to find the right combination of tools is even more challenging. Obviously, finding suitable tools that complete the analysis of microbiome data can be time-consuming and may even require more high-level programming experiences which some users may be lacking.

The core step in microbiome analysis is the taxonomic classification of the representative sequences and clustering of OTUs (Operational Taxonomic Units). OTUs are pragmatic proxies for potential microbial species represented in a sample. Performing quality control of the sequences prior to taxonomic classification is paramount for the identification of poor-quality reads and residual contamination in the dataset. There are several public tools available for inspecting read quality and filtering the poor-quality reads as well as removing any residue contamination. For example, pre-processing tools such as Seqkit [1], FastQC [2] and BBduk.sh command available in the BBMap package [3] are designed to help investigators review the properties and quality of reads before further downstream analyses. High quality reads coupled with stringent screening and filtering can significantly reduce the number of spurious OTUs.

The most famous microbiome analysis tools integrate different quality control approaches in their pipelines. Mothur [4] for example is well known for its intensive quality filtering of poor sequences before OTU clustering and taxonomy assignment. Quantitative Insight Into Microbial Ecology (QIIME-2), a successor of QIIME-1 [5] (see http://qiime.org/) uses DADA2 [6] to obtain highquality representative sequences before aligning them using MAFFT [7] software. Nevertheless, the most common sequencing error is the formation of chimeric fragments during PCR amplification process [8, 9]. Briefly, chimeras are false recombinants formed when prematurely terminated fragments during PCR process reanneal to another template DNA, thus eliminating the assumption that an amplified sequence may have originated from a single microbial organism. Detecting and removing chimeric sequences is crucial for obtaining quality sequence classification results. Both Mothur and QIIME-2 integrate special tools for chimera removal, specifically UCHIME [10] and VSEARCH [11].

The sequences that pass the filtering process are typically searched against a known reference taxonomy classifier at a pre-determined threshold. Most classifiers are publicly available including the Ribosomal Database Project (RDP) [12], SILVA [13], Greengenes [14], and EzBioCloud [15]. Use of frequently updated databases avoids mapping the sequences to obsolete taxonomy names. In some cases, users may opt to train their custom classifiers using, for example, q2-feature-classifier protocol [14, 15] available in QIIME-2 [16, 17] or use any other suitable method. Overclassification of the representative sequences can result in spurious OTUs, but this can be avoided by applying stringent cut-offs [18].

Frequently, users adopt the default settings of their preferred pipelines. For example, the $97 \%$ threshold typically expressed as 0.03 in Mothur and $70 \%$ confidence level expressed as 0.7 in QIIME-2 are default settings in OTU clustering. The final output of most microbiome analysis pipelines is the OTU table. Typically, the OTU table is the primary input for most downstream analyses leading to alpha and beta diversity information in both Mothur and QIIME. The OTU table is typically a matrix of counts of sequences, OTUs or taxa on a per-sample basis. The quality of data in the OTU table depends primarily on the previous analyses which provide input to the pipeline's subsequent steps. Making biological conclusions from the OTU table alone without reviewing the intermediate output is a high risk that could result in inaccurate conclusions.

In the present paper, we developed an improved microbiome analysis pipeline named iMAP (Integrated Microbiome Analysis Pipeline) that integrates exploratory analysis, bioinformatics analysis, intensive visualization of intermediate and final output, and phylogenetic tree annotation. The implementation of iMAP pipeline is demonstrated using a case study where 360 mouse gut samples are intensively analyzed. Throughout the manuscript, "iMAP pipeline" terminology is used instead of "iMAP" for easy readability.

\section{Methods \\ Workflow}

Code for implementing iMAP pipeline contains bundles of commands wrapped individually in driver scripts for performing exploratory analysis, preprocessing of the reads, sequence processing and classification, OTU clustering and taxonomy assignment, and preliminary analysis, and visualization of microbiome data (Fig. 1). The pipeline transforms the output obtained from major analysis steps to provide data structure suitable for conducting exploratory visualization and generating progress reports.

\section{Implementation}

A detailed guideline for implementing iMAP pipeline is in the README file included in the iMAP repository. It is mandatory that all user data files are placed in the 


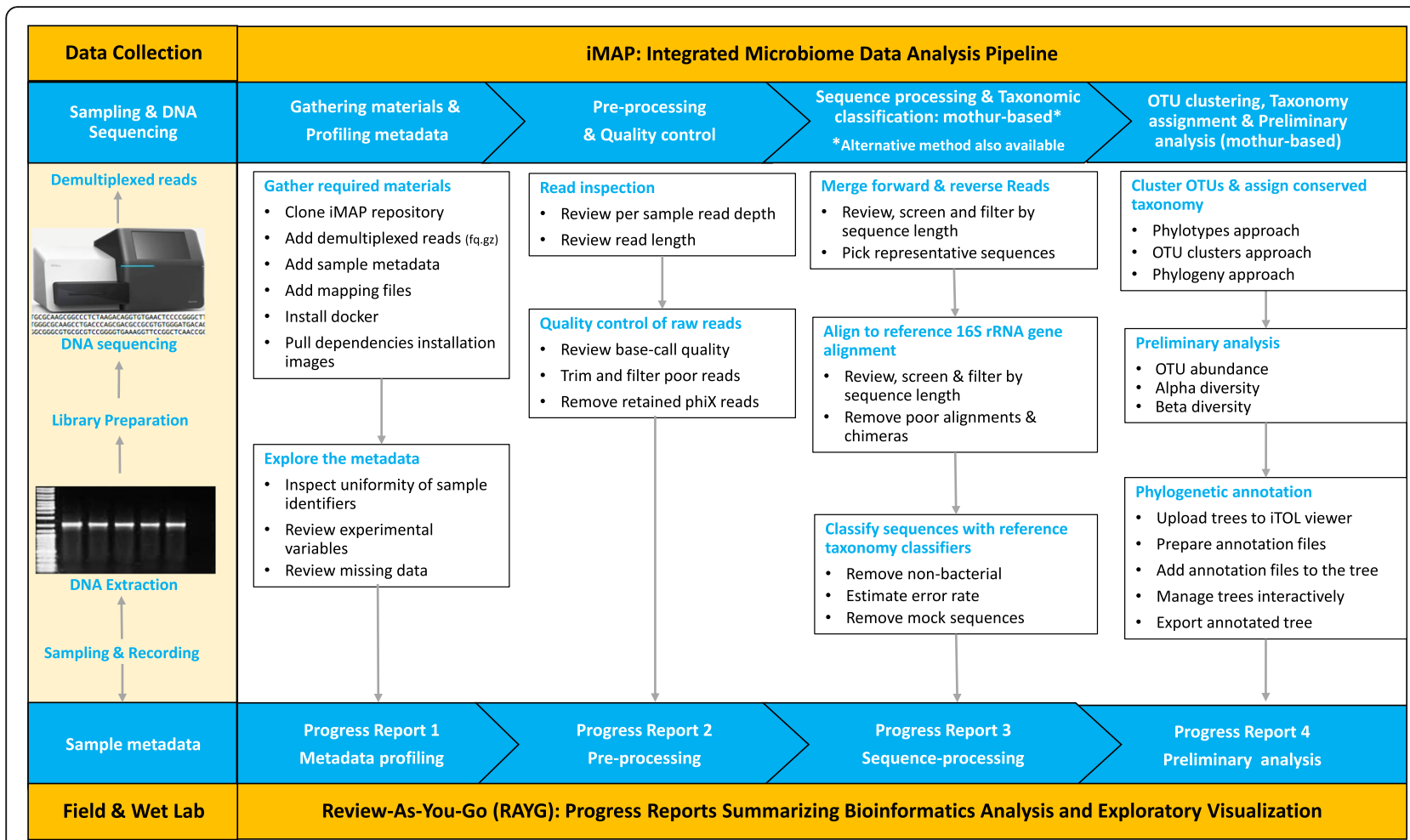

Fig. 1 Schematic illustration of the iMAP pipeline. The required materials including data files, software, and reference databases must be in place before executing the iMAP pipeline. The initial step in the analysis is sample metadata profiling followed by pre-processing and quality checking of demultiplexed 165 read pairs which are then merged, aligned to reference alignments, classified then assigned conserved taxonomy names. Output from each major step is transformed, visualized, and summarized into a progress report

designated folders and must remain unaltered throughout the entire analysis.

\section{Robustness, reproducibility, and sustainability}

Ability to reproduce microbiome data analysis is crucial. Challenges in robustness and reproducibility are accelerated by lack of proper experimental design, the complexity of experiments, constant updates made to the available pipelines, lack of well-documented workflows, and relying on inaccessible or out-of-date codes. The pre-release version of iMAP described in this manuscript (iMAP v1.0) is at the preliminary phase, and perhaps it lacks significant reproducibility aspects compared to the modern bioinformatics workflow management systems such as Nextflow [19], NextflowWorkbench [20], or Snakemake [21]. In its current state, users will be able to follow the guideline presented in the README file and reuse the associated code interactively, including nested bash and visualization scripts to realize similar results. In an effort to ensuring that the iMAP pipeline is reproducible, portable, and shareable, we created Docker images that wrangle the dependencies including software installation and different versions of $\mathrm{R}$ packages. Using Docker images makes it easier for users to deploy the iMAP and run all analyses using containers. Instructions on how to work with Docker are available in the README file. The iMAP pipeline also comes with both mothur and QIIME2 Docker images for the classification of the 16S rRNA gene sequences.

Future sustainability and reproducibility of iMAP depend highly on the use of a well-established workflow management system to provide a fast and comfortable execution environment, which will probably increase the usability as well. A long-term goal is to automate most of the interactive steps and integrate the pipeline with a code that defines rules for deploying across multiple platforms without any modifications.

\section{Bioinformatics analysis}

The iMAP pipeline is intended to be executed interactively from a command line interface (CLI) or from a Docker container CLI to optimize user interaction with the generated output. A detailed guideline is provided in the README file of the iMAP pipeline. Most of the analysis run at default settings unless altered by the user. By default, the iMAP pipeline uses up-to-date SILVA seed classifiers [13] for mothur-based taxonomy assignments or Greengenes classifiers [14] if using QIIME2 pipeline. The SILVA seed and Greengenes databases are relatively small compared to the SILVA NR version which is available for both mothur and QIIME2. Users need to be 
aware that the larger a dataset is, the more memory (RAM) the system requires. Users may opt to use their preferred classifiers and make a small modification in the sequence classification script. Instructions to do so are available in the README file. We are aware that some microbiome experiments do sequence a mock community to help in measuring the error rate due to biases introduced in PCR amplification and sequencing. The mock community sequences are removed automatically before OTU clustering and taxonomy assignment. However, the group name(s) of the mock samples is required. By default, the iMAP removes two groups named Mock and Mock2. Instruction to replace the mock group names is available in the README.

\section{Data transformation and preliminary analysis}

The final output of most microbiome analysis pipelines is the OTU table, which is typically a matrix of counts of sequences, the observations, i.e. OTUs or taxa on a persample basis. The OTU tables are transformed into data structures suitable for further analysis and visualization with R [22]. Most of the analyses and visualization are executed via the RStudio IDE (integrated development environment) [23]. We understand that different investigators prefer different analysis types based on the hypotheses under question. In the following section, we used a case study to demonstrate the application of the iMAP pipeline and the exploratory visualization that provides an insight into the results.

\section{Phylogenetic annotation with iTOL}

We specifically chose a phylogenetic-based annotation with iTOL (integrative Tree Of Life) [24] to be part of the iMAP pipeline as a model for displaying multivariate data in easily interpretable ways. Briefly, phylogenetic annotation of the groups (samples) or taxa requires a pre-built tree such as Newick tree. Fortunately, in both mothur and QIIME2, there are methods for producing Newick trees where samples are clustered using the UPGMA (Unweighted Pair Group Method with Arithmetic Mean). The annotation is done interactively by first uploading the tree into the iTOL tree viewer and then adding prepared plain text annotation files on top of the tree. We advise users to get an overview of different videos, tutorials, and functions available at the iTOL site to understand the details involved in the annotation process.

\section{Application}

\section{Reproducible case study}

Here we use a case study to demonstrate step-by-step how to use iMAP to analyze microbiome data. We use a dataset from a published microbiome study to demonstrate the implementation of the iMAP pipeline. Using published data enables users to see the added value, such as the metadata profiling, preprocessing of reads, extended visualization, and generation of the progress report at every major analysis step. Review of these reports facilitates making an informed decision on whether to proceed or terminate the analysis and make more changes to the experiment.

\section{Preamble}

In 2012 Schloss et al. [25] published a paper in Gut Microbes journal entitled "Stabilization of the murine gut microbiome following weaning". In this study, 360 fecal samples were collected from 12 mice $(6$ female and 6 male) at 35 time points throughout the first year. Two mock community samples were added in the analysis for estimating the error rate. The mouse gut dataset was chosen because it has been successfully used in several studies for testing new protocols and workflows related to microbiome data analysis [26, 27].

\section{Raw data}

The demultiplexed paired-end 16S rRNA gene reads generated using Illumina's MiSeq platform were downloaded from http://www.mothur.org/MiSeqDevelopmentData/StabilityNoMetaG.tar. These reads were the result of amplification of region four (V4) of the 16S rRNA gene. Sample metadata file describing the major features of the experiment and the associated variables was manually prepared. Mapping files, that link paired-end sequences with the samples and design files that linked sample identifiers to individual experimental variables were extracted from the metadata file in a format compatible with Mothur (Additional file 1). Installation of software and download of required reference databases was done automatically. All required materials were placed in the designated folders precisely as described in the guideline and verified using a check file script.

\section{Metadata profiling}

Metadata profiling was done as part of exploratory analysis to specifically explore the experimental variables to help in planning the downstream analysis and find out if there were any issues such as missing data. The sample identifiers were inspected and uniformly coded to facilitate sorting across multiple analytical platforms and for better visualization and uniform labeling of the axes.

\section{Sequence pre-processing and quality control}

Read pre-preprocessing included (i) general inspection using seqkit [1] software to provide basic descriptive information about the reads including data type (DNA or RNA), read depth and read length, (ii) assessing the base call quality using FastQC [2] software, and (iii) trimming and filtering poor reads and removing any retained phiX control reads using BBDuk tool from BBMap [28] 
package. The quality of altered reads was again verified by re-running the FastQC software. The FastQC output was summarized using MultiQC [29] software.

\section{Sequence processing and classification}

This case study uses mothur-based functions to process and classify the representative sequences. The iMAP code also includes a batch script for analyzing the sequences using QIIME2. Preprocessed paired-end reads were merged into more extended sequences then screened to match the targeted V4 region of the $16 \mathrm{~S}$ rRNA gene. The pipeline generated the representative sequences and aligned them to the SILVA-seed v132 rRNA reference alignments [30] to find the closest candidates. Post-alignment quality control involved repeating the screening and filtering the output by length and removal of poor alignments and chimeric sequences. All non-chimeric sequences were searched against SILVA-seed classifiers at $80 \%$ identity using a k-nearest neighbor consensus and Wang approach precisely as described in the Mothur MiSeq SOP tutorial [24].

Additional quality control was done automatically using 'remove.lineage' function run within mothur to remove any non-bacterial or unknown sequences before further analysis. Briefly, by default, the pipeline classified the sequences using SILVA seed taxonomy classifier. If the classifier did not find a match in the database, it grouped the unclassified sequences into 'unknown' category. The iMAP code was set to remove all undesirable matches including the unknown and any sequences classified to nonbacterial lineages such as eukaryotes, chloroplast, mitochondria, viruses, viroid and archaea. The sequencing error rate was then estimated using sequences from the mock community. Finally, after error rate estimation all mock sequences were removed from further analysis.

\section{OTU clustering and conserved taxonomy assignment}

We used a combination of phylotype, OTU-based and phylogeny methods to assign conserved taxonomy to OTUs. Briefly, in phylotype method, the sequences were binned into known phylotypes up to genus level. In the OTU-based method, all sequences were binned into clusters of OTUs based on their similarity at $\geq 97 \%$ identity, and precision and FDR were calculated using the opticlust algorithm, a default mothur function for assigning OTUs. The phylogeny method was used to generate a tree that displayed consensus taxonomy for each node. The output from phylotype, OTU-based and phylogeny methods was manually reviewed, de-duplicated and integrated to form a complete OTU taxonomy output.

\section{Data transformation and preliminary analysis}

We prepared data structures for further analysis and visualization with $\mathrm{R}$ packages executed via RStudio IDE. In summary, the preliminary analysis included measuring diversity in community membership using Jaccard dissimilarity coefficients based on the observed and estimated richness. The diversity in community structure across groups was determined using Bray-Curtis dissimilarity coefficients. The Bray-Curtis dissimilarity coefficients were further analyzed using ordination methods to get a deeper insight into the sample-species relationships. Included in the ordination-based analysis were: (i) Principal Component Analysis (PCA), (ii) Principal Coordinate Analysis (PCoA or MDS) and (iii) Non-Metric Dimensional Scaling (NMDS). Scree plot was used to find the best number of axes that explained variation seen on PCA plots while PCoA loadings and goodness function in vegan [31] was used to generate values for plotting observations into ordination space. Shepard plot was used to compare observations from original dissimilarities, ordination distances and fitted values in NMDS.

\section{Phylogenetic annotation}

Phylogenetic annotation was done using iTOL tree viewer [24] interactively. To see how the samples clustered together we uploaded mothur-based Newick tree generated from the Bray-Curtis dissimilarity distances into the iTOL tree viewer. We then added on top of the tree three iTOLcompatible annotation files prepared manually to specifically include selected output, including species richness, diversity and relative abundances at phylum-level.

\section{Results}

\section{Metadata profiling}

Preliminary analysis of the metadata (Additional file 1: Sheet 1) was done to explore the experimental variables and find out any inconsistency or missing values. The results were automatically summarized into a web-based progress report 1 (Additional file 2). The main variables studied were sex (female and male), time range (early and late) grouped based on days-post-weaning (DPW) (Fig. 2). Reviewing the report enabled us to inspect the input data, find the inconsistency in sample coding, and missing data. Before further analysis, the sample identifiers were uniformly re-coded to six figures, e.g., F3D1, F4D11, M4D145 to F3D001, F4D011, M4D145, respectively. In the subsequent analyses, we defined the numeric categoric variables (DPW) as factors and coded it uniformly as shown in DayID column in the metadata file (Table 1).

\section{Read pre-processing and quality control}

Pre-processing results were automatically summarized into a web-based progress report 2 (Additional file 3). The whole dataset contained 3,634,461 paired-end reads. The original FastQC results showed a minimum Phred score $(\mathrm{Q})$ near 10 and trimming poor quality reads at the default settings $(\mathrm{Q}=25)$ and removal of phiX contaminations resulted into high quality reads (Fig. 3). 


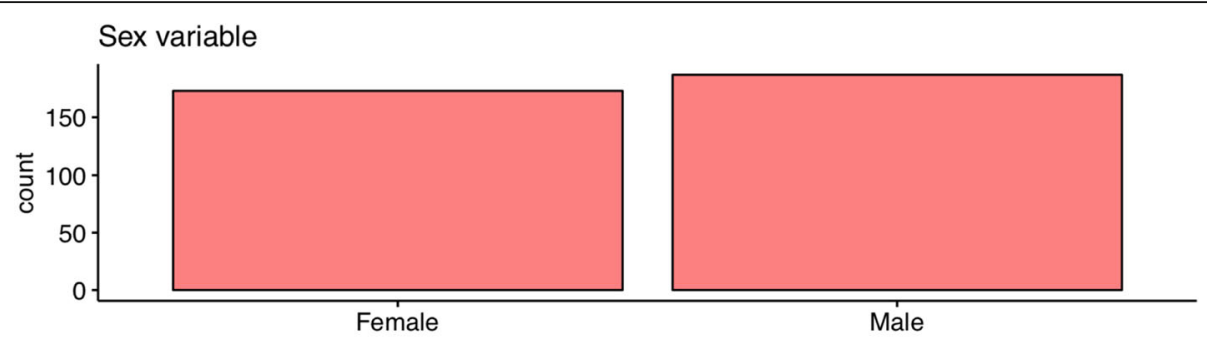

Sex of animal
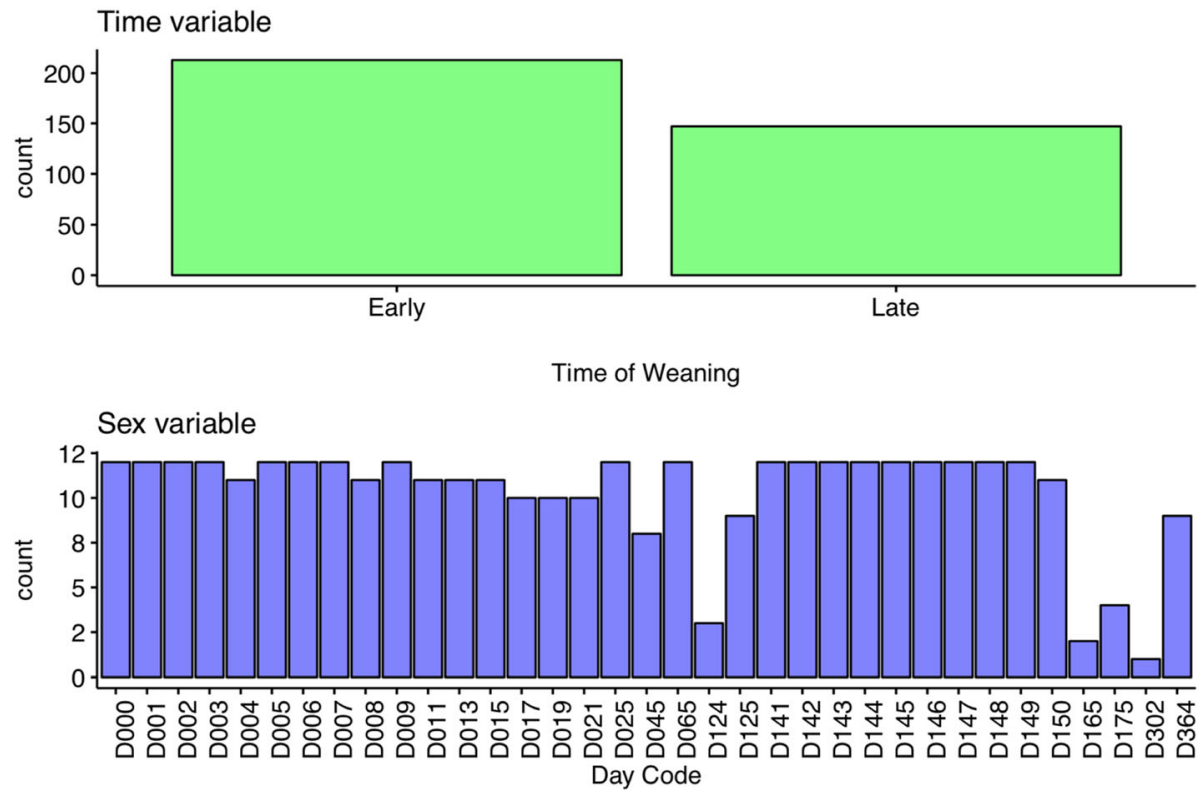

Fig. 2 Frequency of categorical variables. The sex and time variables contain two levels each. The days post weaning (DPW) variable contains 35 levels representing data points where $\mathrm{D}$ stands for the day the data was collected followed by a numeric value specifying the day number within a year, starting from 0 to 364

Distribution of changes was visualized using boxplots, density plots and histogram plots (Fig. 4). The difference between the original and pre-processed reads was very small, barely visible in the distribution plots. Only 2692 $(0.07 \%)$ poor-quality reads were identified in each forward and reverse read (Table 2) indicating that over 99.9\% of the reads qualified for downstream analysis.

\section{Sequence processing and quality control}

The sequence processing and taxonomy assignment results were automatically summarized into a web-based progress report 3 (Additional file 4). This process involved merging 3,631,769 high-quality read pairs to form much longer sequences that were then screened based on their length. Merging the forward and reverse reads

Table 1 Descriptive statistics of the metadata

\begin{tabular}{|c|c|c|c|c|c|c|c|c|}
\hline Variable & q_zeros & p_zeros & q_na & p_na & q_inf & p_inf & type & unique \\
\hline SamplelD & 0 & 0.00 & 0 & 0 & 0 & 0 & character & 360 \\
\hline Group & 0 & 0.00 & 0 & 0 & 0 & 0 & character & 360 \\
\hline Sex & 0 & 0.00 & 0 & 0 & 0 & 0 & character & 2 \\
\hline Time & 0 & 0.00 & 0 & 0 & 0 & 0 & character & 2 \\
\hline DPW & 12 & 3.33 & 0 & 0 & 0 & 0 & integer & 35 \\
\hline DaylD & 0 & 0.00 & 0 & 0 & 0 & 0 & character & 35 \\
\hline Description & 0 & 0.00 & 0 & 0 & 0 & 0 & character & 1 \\
\hline
\end{tabular}

Key: $q \_z e r o s$ quantity of missing data, $p \_z e r o s$ percentage of missing data, $q \_n a$ quantity of NA, $p \_n a$ percentage of NA, $q \_$inf quantity of infinite values, $p \_i n f$ percentage of infinity values, type factor, character, integer or numeric, unique frequency of the values 


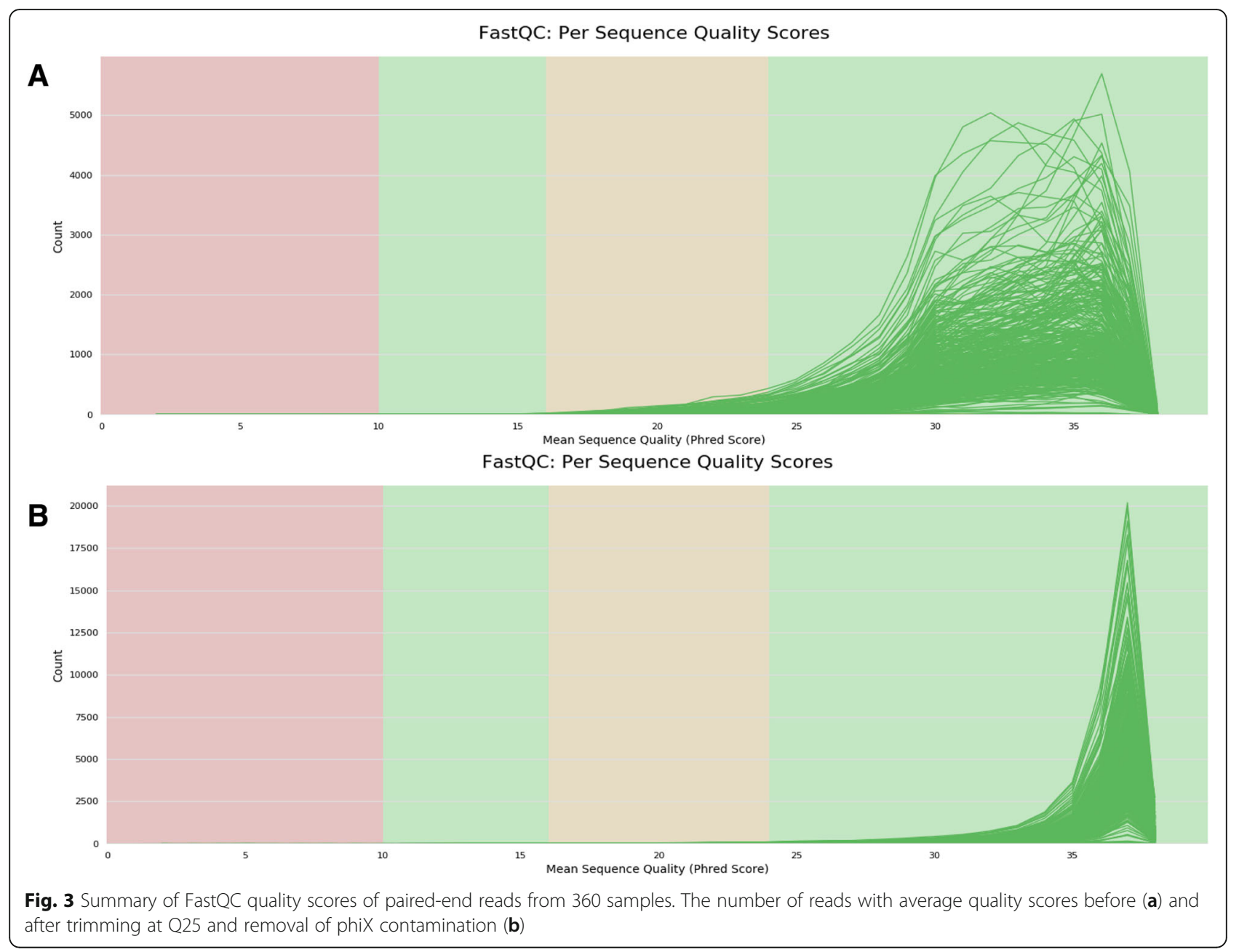

resulted in sequences with 250 nucleotides (Fig. 5a). The 250-nucleotide sequence length is perfectly in-line with the targeted V4 region of the 16S rRNA gene. Most of the overlap fragments were 150 nucleotide long (Fig. 5b) and had mostly zero mismatches (Fig. 5c). Representative sequences (non-redundant) were then searched against SILVA rRNA reference alignments [13] to find the closest $16 \mathrm{~S}$ rRNA gene candidates for downstream analysis. The query length (Fig. 5d) and alignment length (Fig. 5e) showed a high percent identity mostly around 90 and 100\% identity (Fig. 5f). Post-alignment quality control which involved removing poor alignments and chimeric sequences yielded 2,934,726 clean sequences for downstream analysis.

\section{Sequence classification}

All 2,934,726 non-chimeric sequences were searched against Mothur-formatted SILVA-bacterial classifiers at 80\% identity using a k-nearest neighbor consensus and Wang approach as described [20, 21]. The error rate estimated after removing any remaining non-bacterial sequences was $0.00047(0.047 \%)$. Removal of mock community finalizes sequence processing and quality control. Tabular and graphical representation showed a slight alteration of the number of processed sequences (Table 3, Fig. 6).

\section{OTU clustering and taxonomy assignment}

OTU and taxonomy results including preliminary analysis were automatically summarized into a web-based progress report 4 (Additional file 5). Clustering of 2,920, 782 clean sequences into OTUs and assigning taxonomy names was done using a combination of phylotype, OTU-based and phylogeny methods as described in mothur platform [32, 33]. Taxonomy assignment in OTU-based method is by default optimized using opticlust algorithm [34]. This algorithm yielded high-quality results with high precision and low FDR $\leq 0.002$ (Table 4)

\section{OTU abundance and preliminary analysis}

The phylotype method yielded 197 OTUs at genus level while 11,257 OTU clusters were generated by the OTUbased method at $97 \%$ identity. The phylogeny method generated 58,929 tree nodes which were taxonomically classified at $97 \%$ identity. As part of reviewing the 


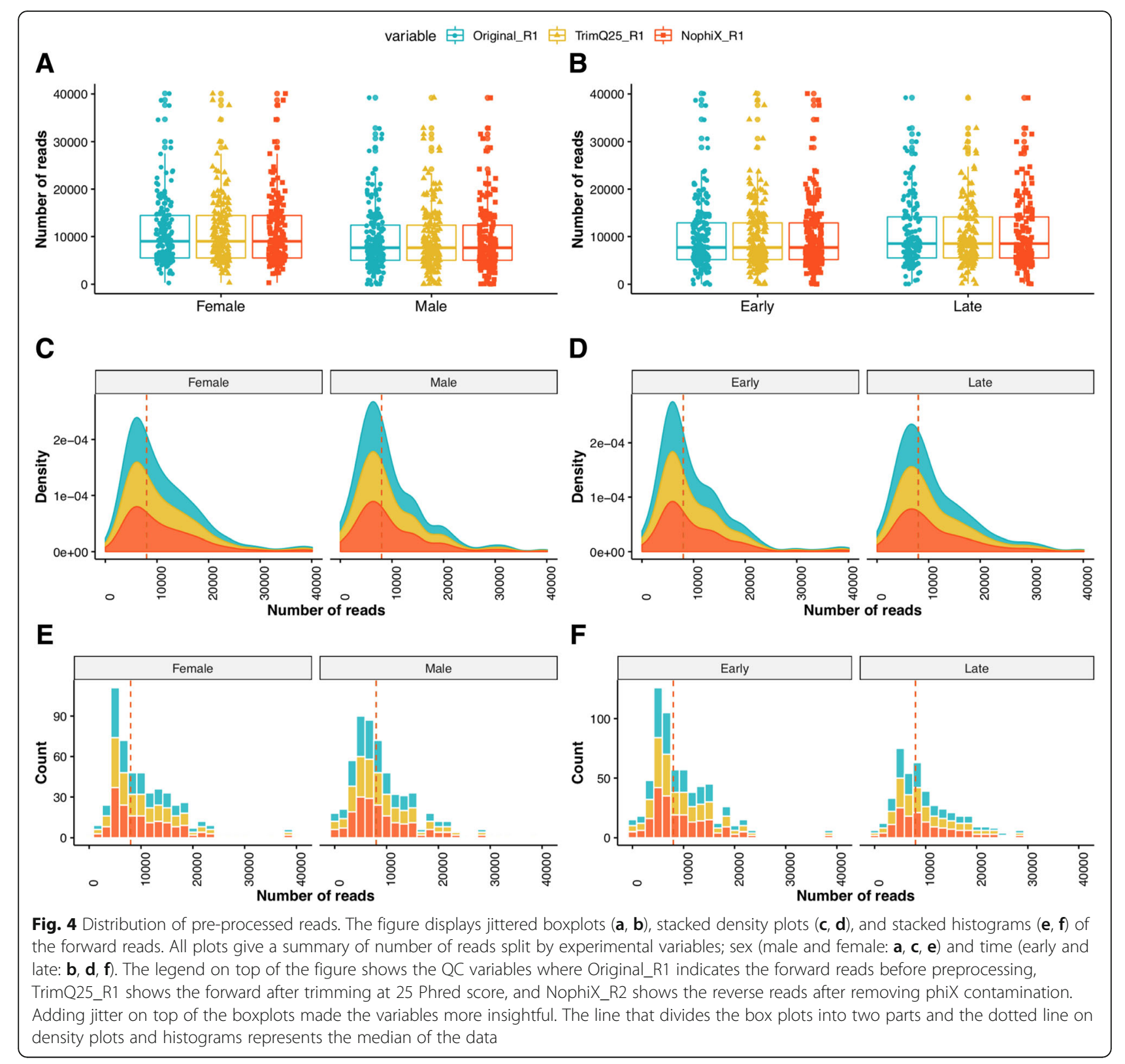

Table 2 Descriptive statistics of the pre-processed reads and total count from all samples

\begin{tabular}{|c|c|c|c|c|c|c|}
\hline Statistics & Original_R1 & TrimQ25_R1 & NophiX_R1 & Original_R2 & TrimQ25_R2 & NophiX_R2 \\
\hline Min. & 14 & 14 & 14 & 14 & 14 & 14 \\
\hline 1st Qu. & 5368 & 5365 & 5365 & 5368 & 5365 & 5365 \\
\hline Median & 8001 & 7996 & 7996 & 8001 & 7996 & 7996 \\
\hline Mean & 10,096 & 10,089 & 10,088 & 10,096 & 10,089 & 10,088 \\
\hline 3rd Qu. & 13,636 & 13,630 & 13,630 & 13,636 & 13,630 & 13,630 \\
\hline Max. & 40,113 & 40,077 & 40,077 & 40,113 & 40,077 & 40,077 \\
\hline Total reads & $3,634,461$ & $3,631,940$ & $3,631,769$ & $3,634,461$ & $3,631,940$ & $3,631,769$ \\
\hline
\end{tabular}



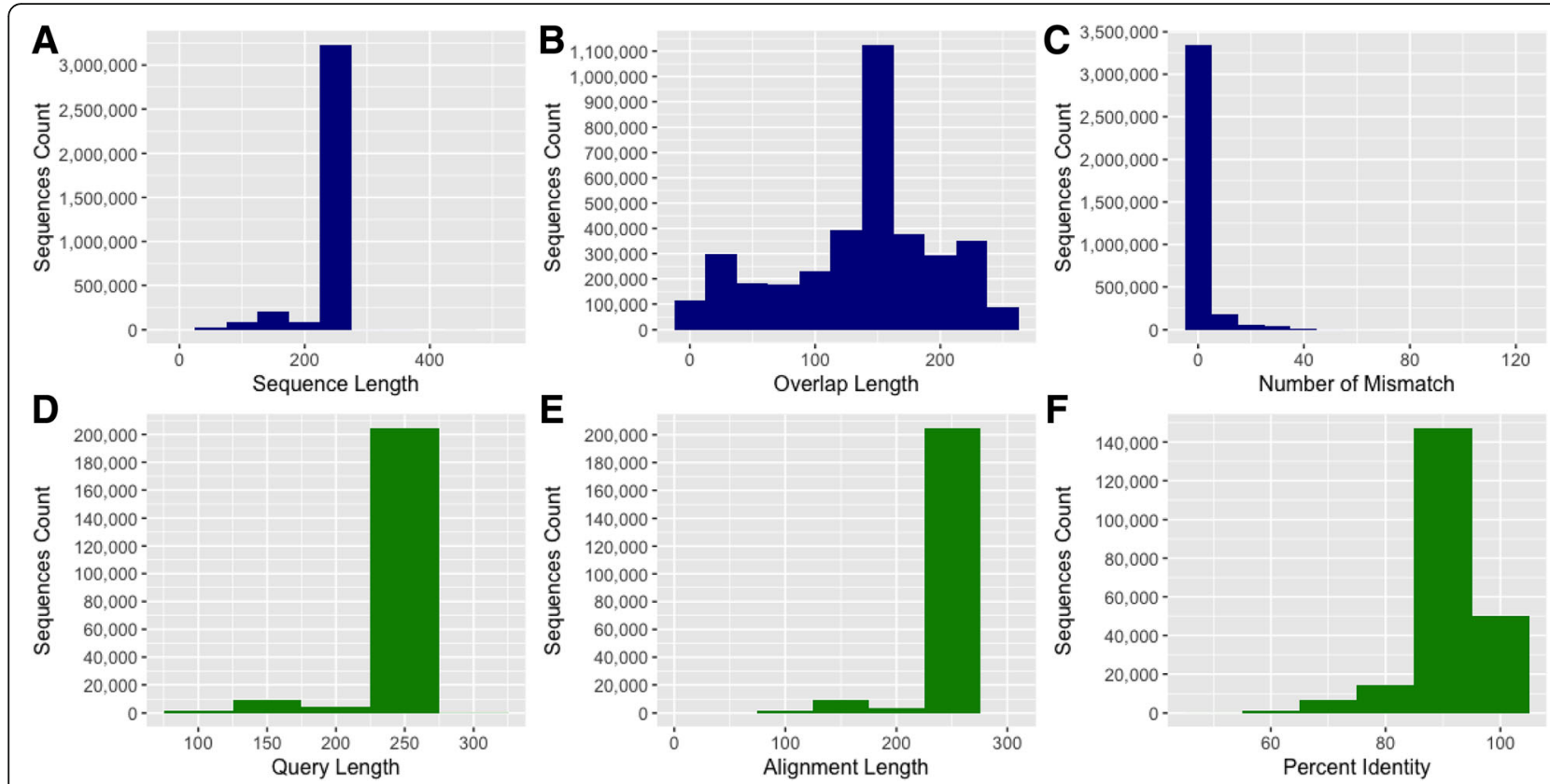

Fig. 5 Features of the assembled and aligned sequences. Merging the forward and reverse reads resulted in sequences with 250 nucleotides (a). The 250-nucleotide sequence length is perfectly in-line with the targeted V4 region of the 16S rRNA gene. Most of the overlap fragments were 150 nucleotides long (b) and had zero mismatches (c). The query length (d) and alignment length (e) showed a high percent identity at 90 and $100 \%(\mathbf{f})$

intermediate results, we compared the taxonomy results across the three classification methods. A high redundancy rate was revealed where different sequences were assigned to same lineages at different percent identity, ranging from 97 to $100 \%$, and had significantly inflated the number of OTUs, particularly in the OTU-based and phylogeny methods. We used the interactive Venn diagram viewer $[35,36]$ to show all possible logical relationships between the three classification methods. Briefly, the list of lineages or the taxa names was uploaded as input. The output was a tabulated textual output indicating the taxonomy lineages or taxon names that were in each intersection or unique to a specific method. Additionally, a graphical output showing the number of elements in each method in the form of symmetric Venn diagrams was generated (Table 5, Fig. 7).

\section{Alpha diversity analysis \\ Species accumulation}

The number of new species added as a function of sites sampling effort was determined using four different accumulator functions as described in the vegan package [31], i.e. exact, random, collector and rarefaction (Fig. 8). Typically, the exact, random, and rarefaction methods calculate standard error bars which can guide investigators to determine which one to choose. Additionally, we used the iNEXT package [37], which enabled us to demonstrate the plotting of rarefaction and extrapolation of species diversity based on sample-size and sample coverage (see details in Additional file 5).

\section{Species richness and diversity}

Estimated and observed species richness were determined using Chao and Sobs calculators, respectively

Table 3 Descriptive statistics of processed sequences

\begin{tabular}{|c|c|c|c|c|c|c|c|}
\hline Statistics & Original & Screened & Aligned & Denoised & NonChimeric & BacteriaOnly & NoMock \\
\hline Minimum & 14 & 8 & 6 & 6 & 6 & 6 & 6 \\
\hline 1st Quantile & 5365 & 4689 & 4658 & 4658 & 4336 & 4328 & 4328 \\
\hline Median & 7996 & 7052 & 7012 & 7012 & 6590 & 6578 & 6578 \\
\hline Mean & 10,088 & 8819 & 8767 & 8767 & 8119 & 8113 & 8113 \\
\hline 3rd Quantile & 13,630 & 11,937 & 11,870 & 11,870 & 10,740 & 10,733 & 10,733 \\
\hline Maximum & 40,077 & 34,866 & 34,590 & 34,590 & 32,161 & 32,156 & 32,156 \\
\hline Total reads & $3,631,769$ & $3,174,898$ & $3,156,040$ & $3,156,040$ & $2,922,704$ & $2,920,782$ & $2,920,782$ \\
\hline
\end{tabular}




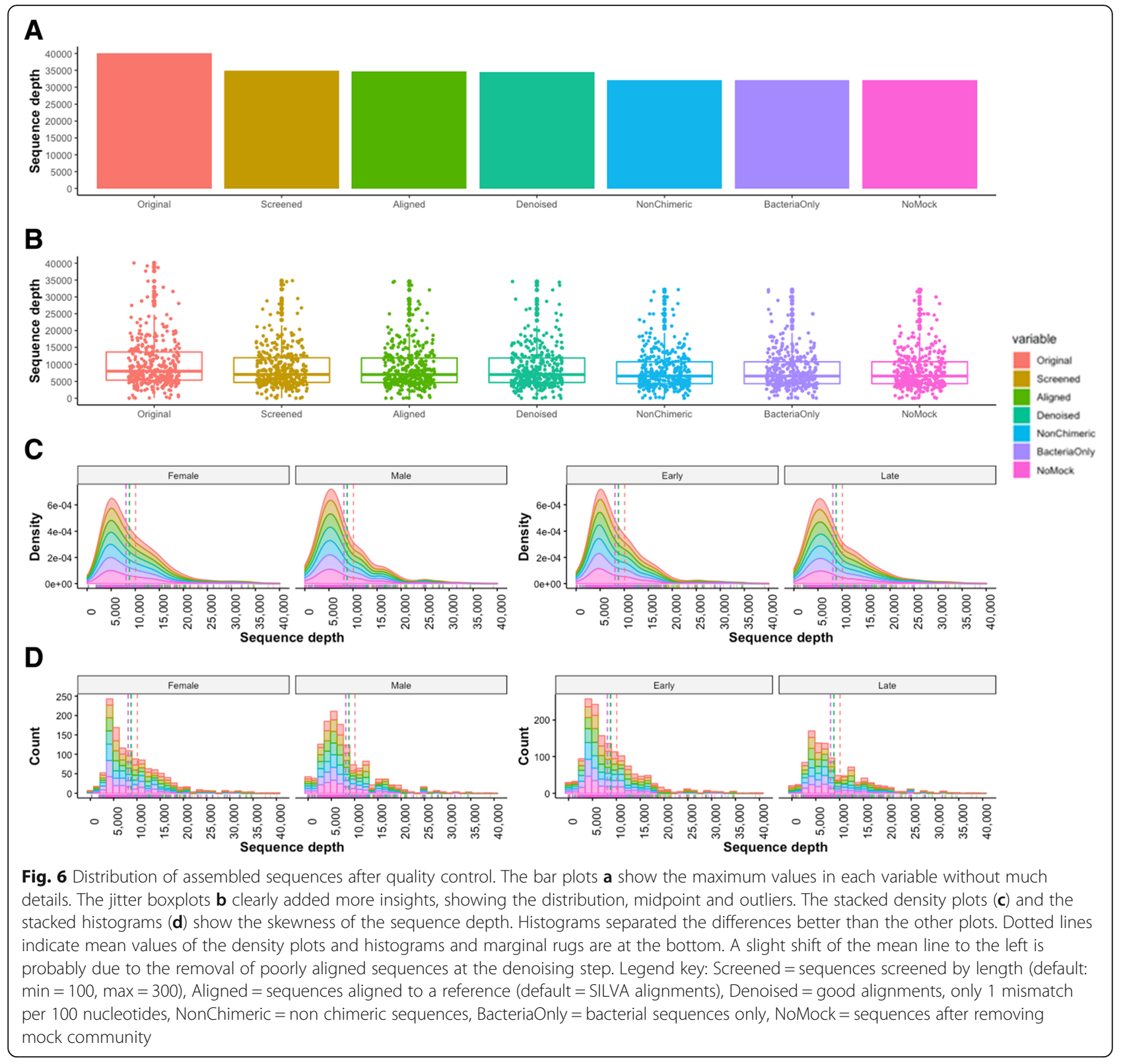

Table 4 Statistical parameters calculated in OTU-based approach

\begin{tabular}{ll}
\hline Parameter & Value \\
\hline Cutoff & 0.2 \\
Sensitivity & 0.998 \\
Specificity & 0.999 \\
PPV & 0.998 \\
NPV & 0.999 \\
Accuracy & 0.999 \\
MCC & 0.997 \\
F1 score & 0.998 \\
\hline
\end{tabular}

(Fig. 9). Three diversity indices including inverse Simpson, Shannon, and phylo-diversity were used to account for the abundance and evenness of species present in the samples.

\section{Beta diversity analysis Clustering and ordination projections}

The difference in microbial community composition across the groups was measured using the raw abundance data and the Bray-Curtis (dis)similarity coefficients. Clustering and ordination projection methods including partition around medoids (PAM) [38], principal component analysis (PCA), principal coordinate analysis (PCoA) and non-metric multidimensional scaling (NMDS) showed 
Table 5 OTU abundance observed in three methods

\begin{tabular}{|c|c|c|c|c|c|c|c|c|}
\hline \multirow[t]{2}{*}{ Method } & \multirow[t]{2}{*}{ Lineages } & \multirow[t]{2}{*}{ Unique lineages } & \multirow[t]{2}{*}{ Unique terms } & \multirow[b]{2}{*}{ Phylum } & \multicolumn{4}{|c|}{ Unique taxon } \\
\hline & & & & & Class & Order & Family & Genus \\
\hline Phylotype & 197 & 197 & 197 & 15 & 24 & 52 & 88 & 139 \\
\hline OTU-based & 11,257 & 197 & 197 & 15 & 24 & 52 & 88 & 139 \\
\hline Phylogeny & 58,929 & 423 & 162 & 10 & 14 & 32 & 54 & 76 \\
\hline
\end{tabular}

similar grouping (Additional file 5). A few components explained the variability of dissimilarity coefficients across the samples in all methods. Silhouette [39] graphical representation validated the consistency within PAM clusters (Fig. 10). Scree plots and data loadings were used to show components that best explained the variation in PCA and PCoA, respectively. Shepard or stress plot confirmed linearity between the original and reduced dimensions in NMDS (see details in Additional file 5).

\section{Phylogenetic relationship of samples}

Bray-Curtis-based Newick tree was uploaded into iTOL (Interactive Tree Of Life) viewer [24] to interactively display unrooted, circular and regular cladograms or phylograms (Fig. 11). Annotation of circular and regular cladograms with various datasets including species richness, diversity indices and relative abundances at phylum-level enabled us to see the diversity across samples.

\section{Discussion}

We developed iMAP, a CLI-based pipeline that streamlines different functionalities from published tools, to collectively unleash the hidden biological knowledge from marker-based microbiome data. The development of this pipeline is guided by the need for a tool that is efficiently executed by a novice user to investigate bacterial communities represented in diverse samples. The iMAP pipeline is integrated with custom functions that generate reports progressively to facilitate RAYG (review-as-you-go), a new approach associated with the pipeline to enable the investigators to review the intermediate output graphically and correct any obvious errors that may lead to wrong or misleading conclusions.

The iMAP pipeline supports a wide range of fundamental analyses for profiling microbial communities present in an environmental sample. Currently, the pipeline operates on demultiplexed data generated by the Illumina platform and performs metadata profiling,

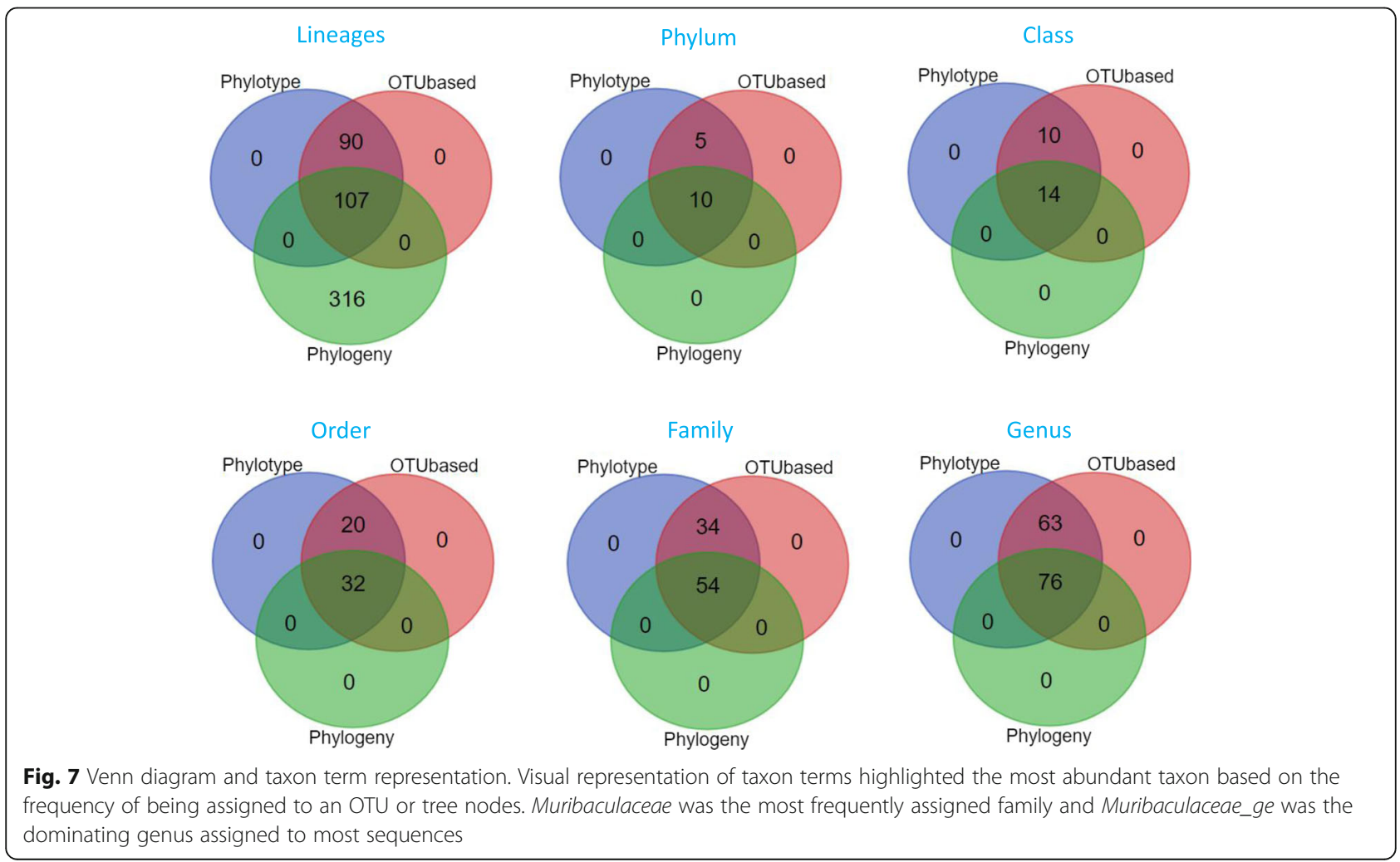




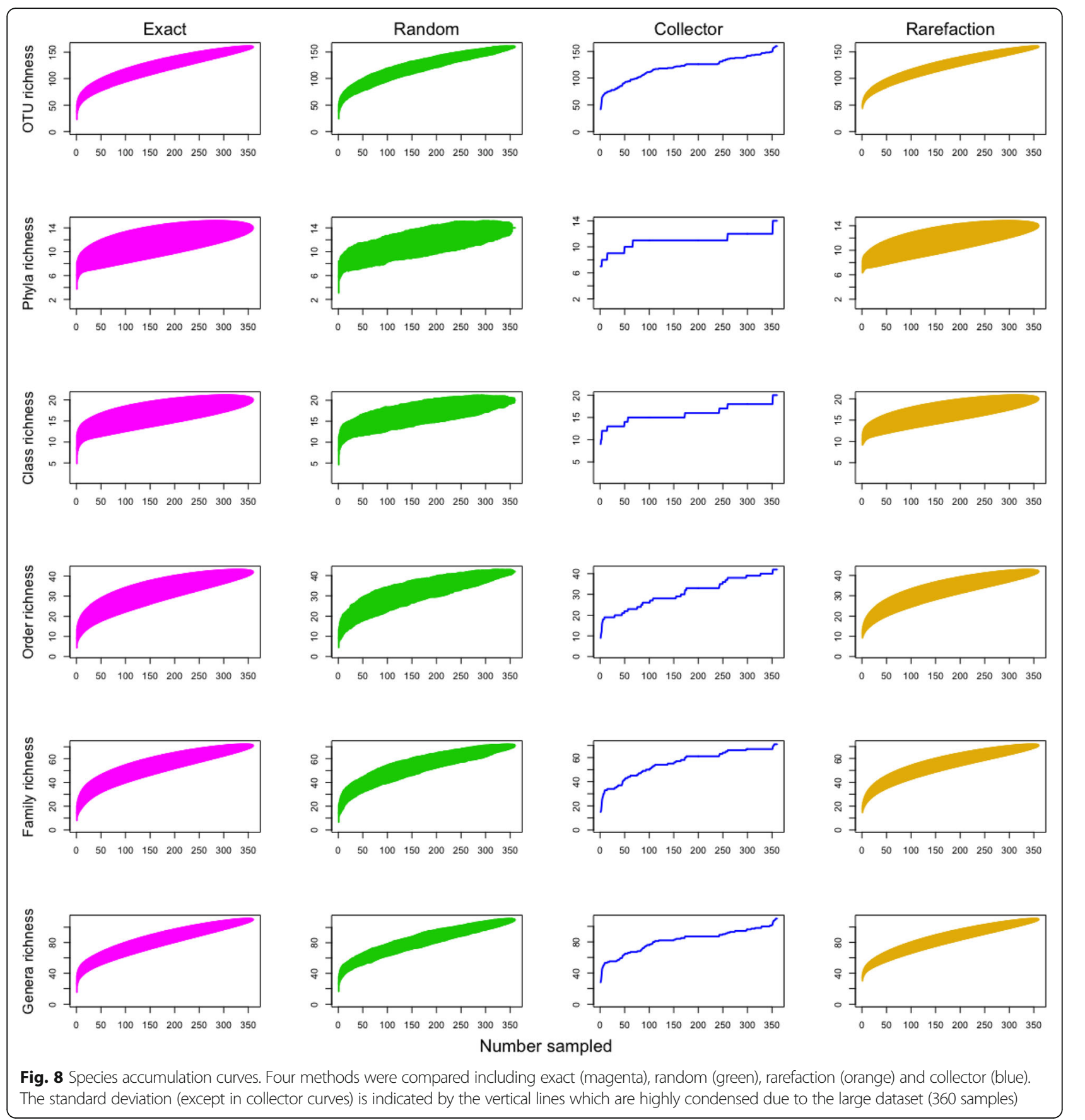

tunable quality filtering, sequence processing, and classification before clustering the representative sequences into OTUs and conserved taxonomy assignment. The idea of implementing RAYG approach at every major step gives the investigators an opportunity of taking care of issues that could result in spurious OTUs and misleading conclusions.

The output generated from the major analysis steps described in the workflow (Fig. 1) is further transformed to simplify exploratory data analysis. We know that some of the intermediate output can be very large to explore but, in such a situation, iMAP uses custom scripts to extract vital information, then transform it for applying in diverse visualization modules. Reproducibility of the iMAP pipeline is ensured by including in the repository the code used for bioinformatics analysis and some custom R-based scripts for generating publication-ready images commonly reported in microbiome-related manuscripts. Users may want to explore the bioinformatics analysis results using methods of their choice or 


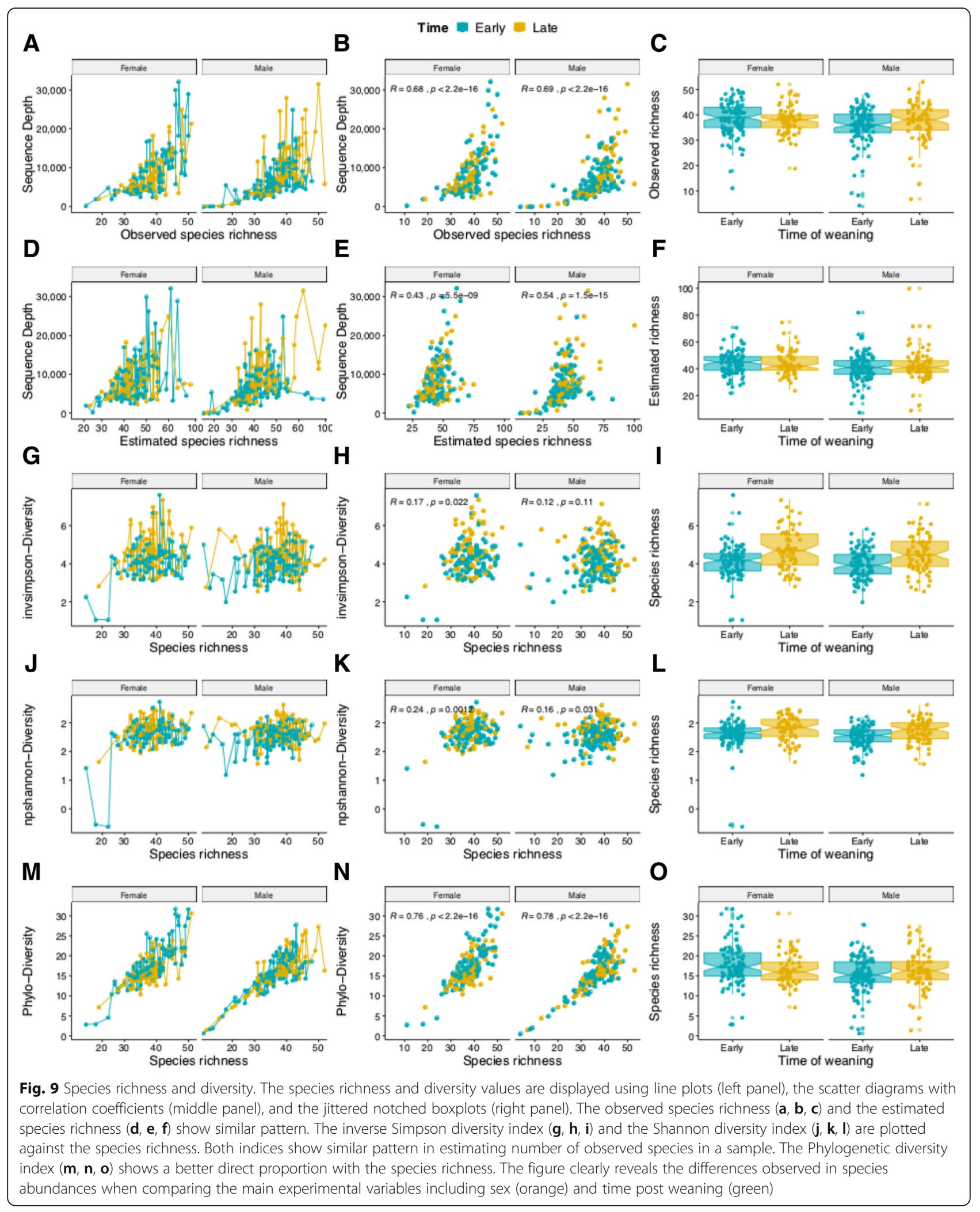




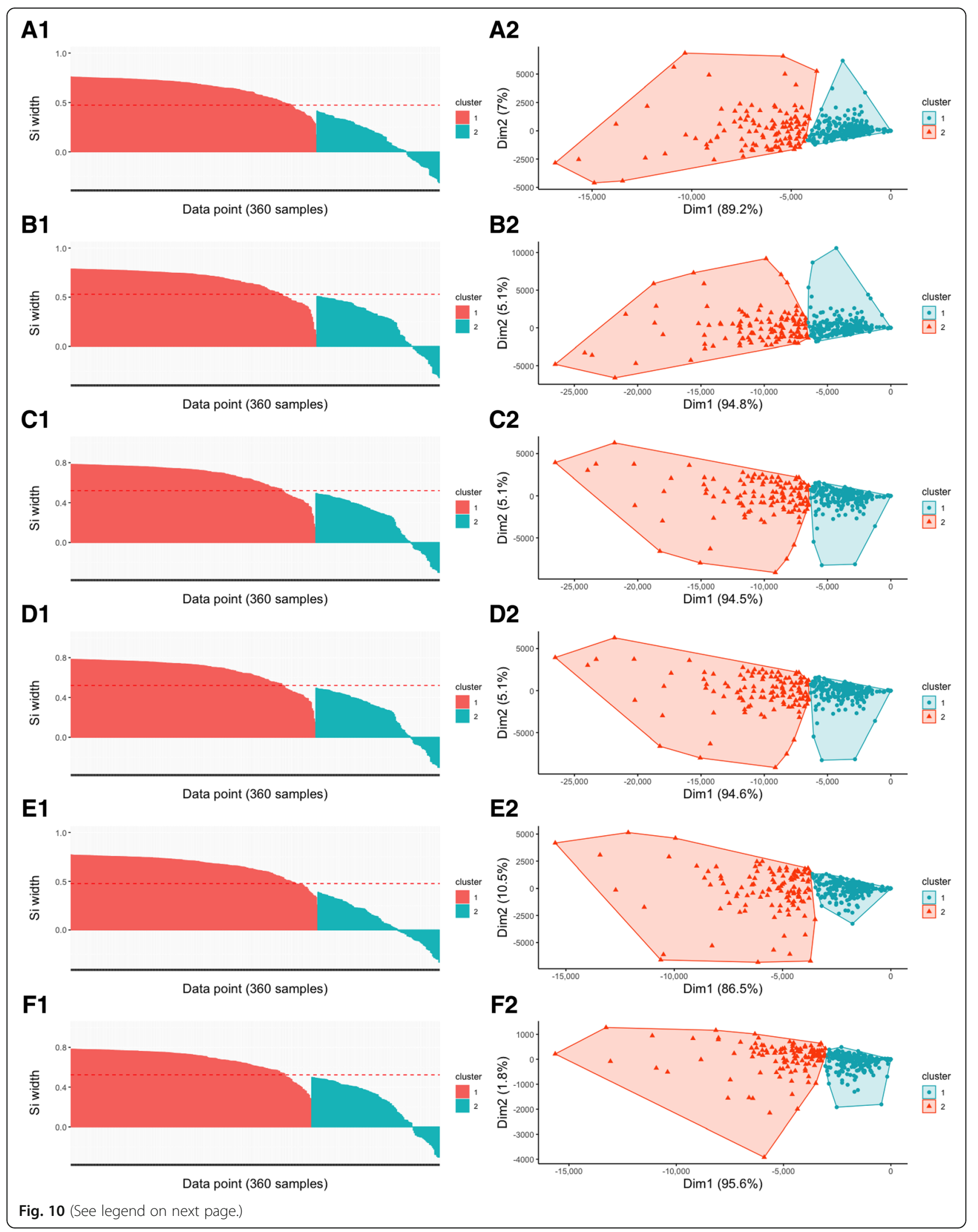


(See figure on previous page.)

Fig. 10 PAM clustering, Silhouette analysis and visualization of clusters. The best number of clusters obtained by PAM clustering method using scaled abundance data for the OTUs (A1), phylum (B1), class (C1), order (D1), family (E1), and genus (F1). Silhouette analysis validates the consistency within PAM clusters where scores with a large Si width (almost 1) indicates high-quality clustering, a small Si width (around 0) means that the observation lies between two clusters while negative values are outliers. The right panel shows the exploratory ordination of the samples onto two axes (Dim1 vs Dim2) based on the abundance of OTUs (A2), phylum (B2), class (C2), order (D2), family (E2), and genus (F2). Similar

samples are close to each other, and dissimilar samples are farther from each other

may modify provided scripts to best describe the different types of data being analyzed. Examples of publication-ready images are presented in each progress report (Additional files 2, 3, 4 and 5). Choice of which type of visualization to use is entirely userdependent and could also depend on how much detail is required.

Post-classification annotation emphasized in this manuscript aim at making the results more accurate. A good example is the number of OTUs identified using different methods where a large was due to the high redundancy rate. Frequently, observed OTU abundance is published unfiltered. While this is not surprising, it can result in misleading conclusions. The fact that there could be some species that contribute disproportionately to the community, it would be sensible to stratify the analyses or performing intensive data annotation afterward. In microbiome data analysis it is normal to see exceptionally abundant species analyzed in the same way as those that are extremely rare. The situation is even worse if the high abundance values are influenced by having lots of redundant values. Adopting focused annotation is the key to achieving appropriate conclusions.

The preliminary analysis workflow included in the pipeline provides several methods for helping users in assessing diversity and statistical comparisons of the variables studied. Species accumulation and rarefaction, for example, provide the best way that allows investigators to figure out whether to continue sampling or whether the data is not enough for drawing a valid conclusion or for estimating normalized sample size for statistical comparisons. Other methods such as heatmap, PAM clustering, and phylogenetic analysis are integrated into the pipeline to find out the relationship of the samples and groups while ordination projections using multivariate statistical techniques such as PCA, PCoA, and

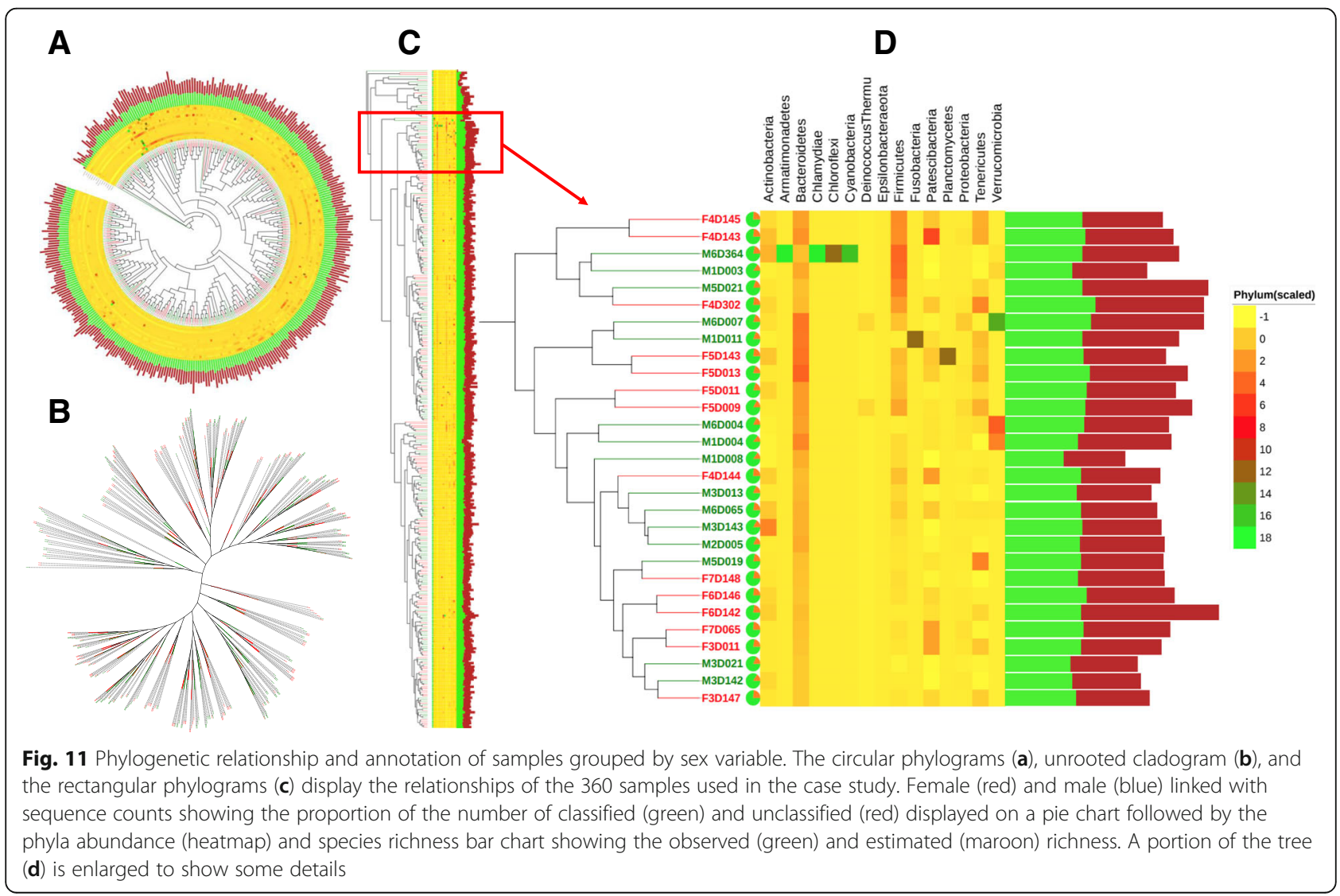


NMDS can be used to identify factors explaining differences among microbial communities. We also provide statistical methods recommended in the mothur platform to compare the experimental variables. AMOVA, HOMOVA, and ANOSIM are among the methods that use $P$-values to determine if the observed differences are statistically significant or are by chance. The Metastats program [40] can be used for detecting differentially abundant microbial communities while the KruskalWallis one-way ANOVA is commonly used to determine if there are statistically significant differences between two or more groups. The mothur-based lefse command modeled after the LEfSe program [41] is an excellent tool for biomarker discovery while the weighted and unweighted UniFrac [42] can be used to compare the samples using their phylogenetic information.

The iMAP pipeline has been successfully tested inhouse using multiple datasets from our bushmeat project (Project \# HDTRA1-16-1-0005). Currently, it is being used to analyze 186 bushmeat samples to characterize the spectrum of microbes present in market bushmeat in Tanzania. The analysis will integrate three groups of variables including three ecosystems (Serengeti, Ruaha, and Selous), two seasons (wet and dry) and two conditions (fresh and processed).

\section{Conclusions}

The iMAP pipeline wraps bioinformatics and visualization tool for generating high-quality user-reviewed microbiome data analysis output. The pipeline integrates read preprocessing, quality control, sequence classification, and OTU taxonomy assignment workflows with data visualization tools to produce high-quality output and diverse visuals for better understanding of complex and multidimensional microbiome data. It also integrates functions that generate reports progressively, emphasizing on the RAYG (review-as-you-go) approach, especially for the in-between process output. Integrating the iMAP with RAYG approach enables the investigators to discover and correct any systematic errors that could lead to misleading conclusions. The multiple statistical methods incorporated in the pipeline guide the investigators to make well-informed decisions and predictions backed by data as well as generating data-driven hypotheses. We believe that users will find this tool broadly useful and adaptable to their microbiome data analysis needs.

\section{Availability and requirements}

Project name: e.g. iMAP

Project home page: https://github.com/tmbuza/iMAP

Operating system(s): Platform independent if using the associated Docker images. Mac OS X or Linux CLI if run outside Docker images.
Programming language: Bash, R 3.5, Perl, Python.

Other requirements: Mothur v1.42 or higher, QIIME2 v2019, Java JDK, Chrome, Firefox, and Safari web browser. License: MIT

Any restrictions to use by non-academics: None.

\section{Additional files}

Additional file 1: Format of input files. Includes sample-metadata mapping (sheet 1), sample-read-file mapping in mothur-format (sheet2), and sample-variable mapping (sheet 3, 4 and 5). (XLSX 69 kb)

Additional file 2: Metadata profiling report generated automatically by the iMAP to provide a summary of the samples and the associated metadata. This report is the initial step in the RAYG (review-as-go) process. The report also displays the R-commands that demonstrates how to reproduce the report. The pipeline is set to automatically save the output in the "reports" folder as "report1_metadata_profiling.html". (HTML $953 \mathrm{~kb})$

Additional file 3: Preprocessing report generated automatically by the IMAP to provide a summary of quality control of the reads. The iMAP pipeline automatically saved the output in the "reports" folder as "report2_read_preprocessing.html". (HTML $3463 \mathrm{~kb}$ )

Additional file 4: Sequence processing report generated automatically by the iMAP to provide a summary of the output. The report was automatically saved in the "reports" folder as "report3_sequence_processing.html". (HTML 4205 kb)

Additional file 5: Preliminary analysis report generated automatically by the iMAP to provide a summary of conserved taxonomy assigned to OTUs and the initial analysis of OTUs and taxa data. The preliminary analysis report was automatically saved in the "reports" folder as "report4_preliminary_analysis.html". (HTML 20379 kb)

\section{Abbreviations}

AMOVA: Analysis of Molecular Variance; ANOSIM: Analysis of Similarity; CLI: Command-Line Interface; FDR: False Discovery Rate; GUI: Graphical User Interface; HOMOVA: Homogeneity of Variance; IDE: Integrated Development Environment; iMAP: Integrated Microbiome Analysis Pipeline; MCC: Matthews Correlation Coefficient; NPV: Negative Predictive Value; OTU: Operational Taxonomic Unit; PAM: Partitioning Around Medoids; PCA: Principal Component Analysis; PCoA: Principal Coordinates Analysis; PPV: Positive Predictive Value; PSU-ICS: Penn State University-Institute for CyberScience; RAYG: Review-As-You-Go; UPGMA: Unweighted Pair Group Method with Arithmetic Mean

\section{Acknowledgements}

Microbiome bioinformatics analysis of the case study was conducted using computational resources provided by the Huck Institutes of Life Sciences and the Institute for CyberScience Advanced Cyberinfrastructure (ICS-ACI) at the Pennsylvania State University, University Park, Pennsylvania, USA. We thank Dr. Istvan Albert (Penn State University) for providing office space to the corresponding author, some computational resources and advice on structuring the iMAP code. We thank Catherine Herzog and Maurice Byukusenge (Penn State University) and Joyce Njuguna (International Livestock Research Institute) for testing the pipeline. We also thank three anonymous reviewers whose comments and suggestions helped improve the manuscript.

\section{Authors' contributions}

VK conceived the original idea. TMB developed the iMAP pipeline, performed the bioinformatic analysis of the case study data, and drafted the manuscript. TT, BL, RK, MS intensively tested the pipeline and provided feedback. FS, CT, PG, IMC, JB, and VK reviewed the manuscript and provided critical feedback and helped shape the second version of the manuscript. All authors read and approved the final manuscript. 


\section{Funding}

This work was funded by a grant from the U.S. Department of Defense, Defense Threat Reduction Agency, Biological Threat Reduction Program, Project \# HDTRA1-16-1-0005. The funders had no role in the design of the study and collection, analysis, and interpretation of data, and in writing the manuscript. The views expressed in this article are those of the authors and do not necessarily reflect the official policy or position of the Defense Threat Reduction Agency, Department of Defense, or the U.S. Government.

\section{Availability of data and materials}

The dataset analyzed during the current study as a case study is publicly available at http://www.mothur.org/MiSeqDevelopmentData/ StabilityNoMetaG.tar. The data supporting the results reported in this manuscript is included within the article and its additional files. The autogenerate progress reports (Additional files 2, 3, 4 and 5) are in HTML format and can be viewed using any preferred browser such as Chrome, Safari, Internet Explorer and Firefox. The iMAP repository which also includes the entire code and other requirements can be downloaded from https://github. com/tmbuza/iMAP. The guidelines for implementing iMAP and related updates, are available at: https://github.com/tmbuza/iMAP/blob/master/ README.md. For further inquiries, please contact the corresponding author at tmbuza@microbiome-bioinfo.com.

\section{Ethics approval and consent to participate}

Not applicable.

\section{Consent for publication}

Not applicable.

\section{Competing interests}

The authors declare that they have no competing interests.

\section{Author details}

${ }^{1}$ The Huck Institutes of the Life Sciences, Pennsylvania State University, University Park, State College, PA, USA. ${ }^{2}$ Department of Biochemistry and Molecular Biology, Pennsylvania State University, University Park, State College, PA, USA. ${ }^{3}$ Biosciences eastern and central Africa-International Livestock Research Institute (BecA-ILRI) Hub, Nairobi, Kenya. ${ }^{4}$ Applied Biological and Biosecurity Research Laboratory, Pennsylvania State University, University Park, State College, PA, USA. ${ }^{5}$ Department of Animal Science, Pennsylvania State University, University Park, State College, PA, USA. ${ }^{6}$ Nelson Mandela African Institute of Science and Technology, Arusha, Tanzania. ${ }^{7}$ Sokoine University of Agriculture, Morogoro, Tanzania. ${ }^{8}$ Department of Biology, Pennsylvania State University, University Park, State College, PA, USA. ${ }^{9}$ Present Address: The European Molecular Biology Laboratory (EMBL), Heidelberg, Germany.

Received: 29 January 2019 Accepted: 24 June 2019

Published online: 03 July 2019

\section{References}

1. Shen W, Le S, Li Y, Hu F. SeqKit: a cross-platform and ultrafast toolkit for FASTA/Q file manipulation. PLoS One. 2016;11(10):e0163962.

2. S. Andrews, "FastQC. 2018: https://www.bioinformatics.babraham.ac.uk/ projects/fastqc/",

3. Bushnell B. BBMap short-read aligner, and other bioinformatics tools; 2016

4. Schloss PD, et al. Introducing mothur: open-source, platform-independent, community-supported software for describing and comparing microbial communities. Appl Env. Microbiol. 2009:75(23):7537-41.

5. Kuczynski J, Stombaugh J, Walters WA, Gonzalez A, Caporaso JG, Knight R. Using QIIME to analyze 16S rRNA gene sequences from microbial communities. Curr Protoc Microbiol. 2012;27(1):1E.5.1-1E.5.20

6. Callahan BJ, McMurdie PJ, Rosen MJ, Han AW, Johnson AJ, Holmes SP. DADA2: high-resolution sample inference from Illumina amplicon data. Nat Methods. 2016;13(7):581-3.

7. Katoh K, Standley DM. MAFFT multiple sequence alignment software version 7: improvements in performance and usability. Mol Biol Evol. 2013; 30(4):772-80.

8. Haas B, Gevers D, Earl A, Feldgarden M, Ward D, Giannoukos G, Ciulla D, Tabbaa D, Highlander S, Sodergren E, Methé B, DeSantis T, Petrosino J, Knight R, Birren B. Chimeric $16 \mathrm{~S}$ rRNA sequence formation and detection in sanger and 454-pyrosequenced PCR amplicons. Genome Res. 2011;21(3): 494-504.

9. Yu W, Rusterholtz KJ, Krummel AT, Lehman N. Detection of high levels of recombination generated during $P C R$ amplification of RNA templates. Biotechniques. 2006;40(4):499-507.

10. Edgar RC, Haas BJ, Clemente JC, Quince C, Knight R. UCHIME improves sensitivity and speed of chimera detection. Bioinformatics. 2011;27(16): 2194-200.

11. Rognes T, Flouri T, Nichols B, Quince C, Mahe F. VSEARCH: a versatile open source tool for metagenomics. PeerJ. 2016;4:e2584.

12. Cole JR, et al. The ribosomal database project (RDP-II): sequences and tools for high-throughput rRNA analysis. Nucleic Acids Res. 2005;33(Database issue):D294-6.

13. Yilmaz $P$, et al. The SILVA and 'all-species living tree project (LTP)' taxonomic frameworks. Nucleic Acids Res. 2014:42(Database issue):D643-8.

14. DeSantis TZ, et al. Greengenes, a chimera-checked 16S rRNA gene database and workbench compatible with ARB. Appl Env. Microbiol. 2006;72(7):506972.

15. Yoon SH, Ha SM, Kwon S, Lim J, Kim Y, Seo H, Chun J. Introducing EzBioCloud: a taxonomically united database of $16 \mathrm{~S}$ rRNA gene sequences and whole-genome assemblies. Int J Syst Evol Microbiol. 2017:67:1613-17.

16. Bokulich NA, et al. Optimizing taxonomic classification of marker-gene amplicon sequences with QIIME 2's q2-feature-classifier plugin. Microbiome. 2018;6(1):90

17. Bokulich N, Dillon M, Bolyen E, Kaehler BD, Huttley GA, Caporaso JG. q2sample-classifier: machine-learning tools for microbiome classification and regression. J Open Source Softw. 2018;3(30):934, https://doi.org/10.21105/ joss.00934.

18. Edgar RC. Updating the $97 \%$ identity threshold for 16 s ribosomal RNA OTUs. Bioinformatics. 2018;34(14):2371-5.

19. Di Tommaso P, Chatzou M, Prieto P, Palumbo E, Notredame C. Nextflow: A tool for deploying reproducible computational pipelines [version 1; not peer reviewed]. F1000Research 2015, 4(ISCB Comm J):430 (poster) (https:// doi.org/10.7490/f1000research.1110183.1)

20. Thomer AK, Twidale MB, Guo J, Yoder MJ. NextflowWorkbench: reproducible and reusable workflows for beginners and experts. In: Conference on human factors in computing systems - proceedings; 2016.

21. Köster J, Rahmann S. Snakemake-a scalable bioinformatics workflow engine. Bioinformatics. 2012;28(19):2520-22.

22. R Core Team. R: A language and environment for statistical computing. Vienna: R Foundation for Statistical Computing; 2018. $R$ version 3.5.2. URL https://www.R-project.org.

23. Team RS. RStudio: integrated development for R. Boston: RStudio, Inc.; 2015.

24. Letunic I, Bork P. Interactive tree of life (iTOL) v3: an online tool for the display and annotation of phylogenetic and other trees. Nucleic Acids Res. 2016;44(W1):W242-5.

25. Schloss PD, Schubert AM, Zackular JP, Iverson KD, Young VB, Petrosino JF. Stabilization of the murine gut microbiome following weaning. Gut Microbes. 2012:3(4):383-93.

26. Westcott SL, Schloss PD. De novo clustering methods outperform reference based methods for assigning $16 \mathrm{~S}$ rRNA gene sequences to operational taxonomic units. PeerJ. 2015;3:e1487.

27. Callahan BJ, Sankaran K, Fukuyama JA, McMurdie PJ, Holmes SP. Bioconductor workflow for microbiome data analysis: from raw reads to community analyses. F1000Research. 2016;5:1492.

28. Segata N, et al. BBMap short-read aligner, and other bioinformatics tools. Bioinformatics. 2016;12(1):17-23.

29. Ewels P, Magnusson M, Lundin S, Kaller M. MultiQC: summarize analysis results for multiple tools and samples in a single report. Bioinformatics. 2016:32(19):3047-8.

30. Mothur, "Taxonomy outline," Online publicly available data, 2018.

31. Oksanen J, et al. Vegan: community ecology package. R Packag. 2018;2:5-2.

32. Kozich JJ, Westcott SL, Baxter NT, Highlander SK, Schloss PD. Development of a dual-index sequencing strategy and curation pipeline for analyzing amplicon sequence data on the MiSeq Illumina sequencing platform. Appl Env Microbiol. 2013:79(17):5112-20.

33. P. D. Schloss, "MiSeq SOP," 2018. Available: https://www.mothur.org/wiki/ MiSeq_SOP.

34. Westcott SL, Schloss PD. OptiClust, an improved method for assigning amplicon-based sequence data to operational taxonomic units. mSphere. 2017;2:e00073-17. https://doi.org/10.1128/mSphereDirect.00073-17. 
35. Bardou P, Mariette J, Escudié F, Djemiel C, Klopp C. Jvenn: An interactive Venn diagram viewer. BMC Bioinformatics. 2014;15(1):293.

36. de Peer V. Bioinformatics \& Evolutionary Genomics: An online web-based tool to calculate and draw custom Venn diagrams. (http://bioinformatics. psb.ugent.be/webtools/Venn/). Accessed 12 Jan 2019.

37. Hsieh TC, Ma KH, Chao A. iNEXT: an R package for interpolation and extrapolation in measuring species diversity. Methods Ecol Evol. 2016;7: $1451-56$.

38. Jin X, Han J. K-Medoids clustering. In: Encyclopedia of machine learning and data mining; 2017.

39. Rousseeuw PJ. Silhouettes: a graphical aid to the interpretation and validation of cluster analysis. J Comput Appl Math. 1987;20:53-65.

40. White JR, Nagarajan N, Pop M. Statistical methods for detecting differentially abundant features in clinical metagenomic samples. PLoS Comput Biol. 2009;5(4): 1 1000352.

41. Segata $\mathrm{N}$, et al. Metagenomic biomarker discovery and explanation. Genome Biol. 2011;12(6):R60.

42. Lozupone C, Lladser ME, Knights D, Stombaugh J, Knight R. UniFrac: an effective distance metric for microbial community comparison. ISME J. 2011: 5:169-72.

\section{Publisher's Note}

Springer Nature remains neutral with regard to jurisdictional claims in published maps and institutional affiliations.

Ready to submit your research? Choose BMC and benefit from:

- fast, convenient online submission

- thorough peer review by experienced researchers in your field

- rapid publication on acceptance

- support for research data, including large and complex data types

- gold Open Access which fosters wider collaboration and increased citations

- maximum visibility for your research: over $100 \mathrm{M}$ website views per year

At BMC, research is always in progress.

Learn more biomedcentral.com/submissions 